\title{
Influência do índice de massa corpórea e frequência de atividades físicas no conforto térmico humano: análise estatística de dados de estudo de campo com usuários de escritórios em Florianópolis, SC
}

\author{
Influence of body mass index and frequency of physical \\ activities on human thermal comfort: statistical analysis \\ of field study data with office occupants in Florianópolis, \\ $\mathrm{SC}$
}

\section{Matheus de Cezaro Menegatti Ricardo Forgiarini Rupp Enedir Ghisi}

\section{Resumo}

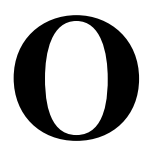

objetivo deste trabalho é verificar a influência do índice de massa corpórea (IMC) e da frequência de atividades físicas na sensação, preferência, conforto e aceitabilidade térmica de pessoas que trabalham em escritórios com ventilação híbrida ou que operam com sistema de ar-condicionado central em Florianópolis, SC. Analisaram-se os resultados de 85 estudos de campo (medições ambientais e aplicação de questionários) em três edificações. Os dados foram separados conforme cada modo de operação e subdivididos em dois subgrupos, um de IMC e outro de frequência de atividades físicas; e submetidos a análises estatísticas com as variáveis de conforto térmico. Observou-se uma tendência de os valores de isolamento da vestimenta diminuírem conforme se aumentam os valores de IMC. Sensação, preferência e conforto térmico mostraram relação significativa com os dois modos de operação, quando levados em consideração os valores de IMC. Em geral, indivíduos com maiores IMCs sentem o ambiente mais aquecido, tendem a preferir ambientes mais resfriados e sentem-se mais confortáveis termicamente do que usuários com menores valores de IMC. Os resultados relacionados à frequência de atividades físicas não se mostraram conclusivos.

Palavras-chave: Conforto térmico. Índice de massa corpórea. Atividade física. Arcondicionado central. Ventilação híbrida. Matheus de Cezaro Menegatti
Universidade Federal de Santa
Catarina
Florianópolis - SC - Brasil

Ricardo Forgiarini Rupp Universidade Federal de Santa Florianópolis - SC - Brasil

Enedir Ghisi

Universidade Federal de Santa Catarina Florianópolis - SC - Brasil

Recebido em 07/11/17 Aceito em 19/03/18

\section{Abstract}

The aim of this study was to verify the influence of body mass index (BMI) and frequency of physical activities on the thermal sensation, preference, comfort and acceptability of people working in offices with hybrid ventilation or operating with a central air-conditioning system in Florianópolis/SC. The results of 85 field studies (environmental measurements and application of questionnaires) were analysed in three buildings. Data were organised according to each mode of operation and subdivided into two subgroups, BMI and frequency of physical activity, and then submitted to statistical analysis with thermal comfort variables. A tendency of decreasing clothing insulation as BMI increased was observed. Thermal sensation, preference and comfort showed a significant relationship with the two modes of operation, when considering BMI values. Overall, individuals with higher BMIs feel warmer, tended to prefer cooler environments and feel more thermally comfortable than users with lower BMI values. Results related to the frequency of physical activities were not conclusive.

Keywords: Thermal comfort. Body mass index. Physical activity. Central air-conditioning. Hybrid ventilation. 


\section{Introdução}

Na área da fisiologia humana, o conceito de zona individual de neutralidade térmica tem sido utilizado para explicar as diferenças entre indivíduos com pesos distintos e também entre outras variáveis, como sexo e idade (KINGMA; FRIJNS; VAN MARKEN LICHTENBELT, 2012). Van Marken Lichtenbelt e Kingma (2013) esclareceram que a zona de neutralidade térmica não é definida pela sensação térmica das pessoas, mas por parâmetros fisiológicos (produção de suor e metabolismo, por exemplo). A zona individual de neutralidade térmica, definida como a faixa de temperatura ambiental na qual a regulação da temperatura corporal é atingida por meio de perda de calor sensível (seco), não envolvendo mudanças no metabolismo ou perdas de calor por evaporação, é influenciada por diversos fatores (metabolismo, idade, sexo, entre outros) e varia entre condições e entre indivíduos (KINGMA; FRIJNS; VAN MARKEN LICHTENBELT, 2012). O limite inferior da zona de neutralidade térmica aponta para temperaturas menores em obesos, quando comparados a pessoas magras (os obesos poderiam precisar de menores temperaturas para estar em conforto térmico, devido ao aumento do isolamento dos tecidos, causado pela maior adiposidade entre os músculos e a pele) (KINGMA; FRIJNS; VAN MARKEN LICHTENBELT, 2012).

Estudos têm sido realizados com o intuito de avaliar o conforto térmico em função das características antropométricas das pessoas. Um dos parâmetros analisados é o peso, comumente associado ao índice de massa corpórea (IMC).

Leites et al. (2013) confirmaram que indivíduos acima do peso apresentam resfriamento evaporativo mais reduzido do que aqueles abaixo do peso.. No entanto, indivíduos abaixo do peso, quando sujeitos a atividades físicas na presença de calor, possuem menor aceitação do ambiente do que aqueles acima do peso. Os autores deixaram claro que o aumento da temperatura corpórea durante a prática de exercícios, e consequentemente o aumento da sensação de calor, está relacionado à condutividade térmica do tecido adiposo, presente em maior quantidade em pessoas acima do peso. Logo, quanto maior a camada de tecido adiposo, maior a preferência por ambientes com temperatura mais baixa.

Claessens-van Ooijen et al. (2006) concluíram que indivíduos magros, quando expostos a ambientes frios por uma hora, tiveram aumento metabólico de cerca de $20 \%$, enquanto indivíduos com maior adiposidade apresentaram aumento metabólico de cerca de 6\%. Wijers, Saris e Van Marken Lichtenbelt (2010) adicionaram os valores de IMC à pesquisa e concluíram que a diferença apresentada no gasto energético está na diferença da espessura da camada de gordura subcutânea e as temperaturas medidas na pele. A partir disso, os autores concluíram que indivíduos obesos possuem maior isolamento térmico devido a maior espessura na camada de gordura, e por isso a perda de calor no corpo é dificultada. Logo, não é necessário grande aumento na geração energética provinda do metabolismo para manter a temperatura constante.

Fadeyi (2014) observou, em seus experimentos com seres humanos, realizados durante o verão em ambiente com sistema de ar-condicionado central, que a sensação térmica de pessoas com sobrepeso foi maior (sentiram-se mais aquecidas) quando comparada com a sensação de pessoas com peso normal. Além disso, os níveis de aceitabilidade térmica, que expressa a aceitação térmica do ambiente pelo indivíduo, foram menores para as pessoas com sobrepeso.

Mozaffarieh et al. (2010) realizaram experimentos com o intuito de investigar a relação de desconforto térmico com extremidades frias considerando diferenças de idade, sexo e IMC em uma população urbana suíça. Os autores concluíram que indivíduos com IMC menor sofreram muito mais desconforto por extremidades frias quando comparados aos do mesmo grupo com IMC superior.

De acordo com Maeda et al. (2005), a produção de calor por meio de calafrios é estreitamente relacionada à proporção de massa magra muscular da pessoa, o que depende da frequência de atividade física (humanos com o hábito diário de exercício têm alto nível de aptidão física e, portanto, maior proporção de massa magra). Outro fator importante a ser considerado é o metabolismo basal, que segundo os autores é o principal responsável pela produção de calor pelo corpo. Dessa maneira, os autores realizaram experimentos com humanos, envolvendo exposições a diferentes temperaturas e a realização de atividade física. Os autores mostraram que pessoas com hábitos de praticar exercícios diários possuíam metabolismo basal mais elevado do que pessoas sedentárias. Assim, esses indivíduos toleraram melhor o frio em comparação a indivíduos com menor metabolismo basal (sedentários), sem ter de aumentar a produção de calor metabólico.

Vecchi (2015), ao analisar indivíduos em clima subtropical no Brasil, chegou à conclusão que a temperatura neutra preferida encontrada para cada grupo de ocupantes, quando separados segundo os valores de IMC foi: $24,6{ }^{\circ} \mathrm{C}$ para indivíduos com “peso normal”; 22,9 ${ }^{\circ} \mathrm{C}$ para indivíduos “acima do 
peso” e 21,9 ${ }^{\circ} \mathrm{C}$ para indivíduos “obesos”, mostrando que conforme os valores de IMC aumentam, diminui a respectiva temperatura neutra preferida.

O objetivo deste artigo é verificar a influência do índice de massa corpórea e frequência de atividades físicas na sensação, preferência, conforto térmico e aceitabilidade térmica de pessoas que trabalham em escritórios com ventilação híbrida ou que operam com sistema de ar-condicionado central na cidade de Florianópolis, SC.

\section{Método}

O foco deste trabalho está na análise estatística de dados previamente coletados sob a ótica de duas variáveis humanas que possivelmente afetam o conforto térmico: IMC e frequência de atividades físicas.

\section{Levantamento dos dados}

Os dados analisados neste trabalho foram obtidos em medições de campo em escritórios, durante os meses de março a outubro de 2014, por uma equipe de pesquisadores do Laboratório de Eficiência Energética em Edificações, e utilizados na tese de Vecchi (2015) e em Vecchi et al. (2017), Rupp et al. (2017) e Rupp e Ghisi (2017).

As medições de campo realizaram-se em três diferentes edificações públicas de escritórios em Florianópolis, SC. Nos edifícios, as salas são compartilhadas entre os trabalhadores. Não serão informados os nomes dos participantes e nem as empresas que participaram do estudo, pois os responsáveis pelas empresas não permitiram. Logo, as edificações denominaram-se por A, B e C. A edificação A é a única a operar com sistema de arcondicionado central durante todo o ano, enquanto as edificações B e C operam com ventilação híbrida (sistema de ar-condicionado e ventilação natural são alternados conforme a preferência dos usuários).

Para o levantamento de dados utilizaram-se dois confortímetros (Figura 1) equipados com sensores para medição da temperatura do ar, umidade relativa do ar, velocidade do ar e temperatura de globo. Em conjunto com os confortímetros também se utilizaram termoanemômetros portáteis (Figura 2) para a medição pontual de temperatura e velocidade do ar. As medições ambientais realizaram-se de acordo com a ASHRAE 55 (AMERICAN..., 2013).

A coleta de dados subjetivos realizou-se por meio da aplicação de um questionário eletrônico que contém seis etapas intercaladas por intervalos de tempo de 20 minutos, com perguntas que buscam respostas pessoais e momentâneas, ou seja, diz respeito ao que a pessoa estava sentindo no momento em que respondeu às perguntas. Por meio do questionário obtiveram-se os seguintes dados dos usuários: vestimentas utilizadas, atividade laboral, frequência de atividades físicas fora do trabalho ("Diariamente”, "Semanalmente” ou "Nunca”), sexo, peso, altura, sensação térmica (escala de sete pontos da ASHRAE: -3 muito frio; 2 frio; -1 levemente com frio; 0 neutro; +1 levemente com calor; +2 calor; +3 muito calor), preferência térmica ("mais aquecido", "assim mesmo" ou "mais resfriado"), conforto térmico (“confortável” ou "desconfortável”) e aceitabilidade térmica ("aceitável” ou “inaceitável”).

\section{Figura 1 - Estação microclimática}

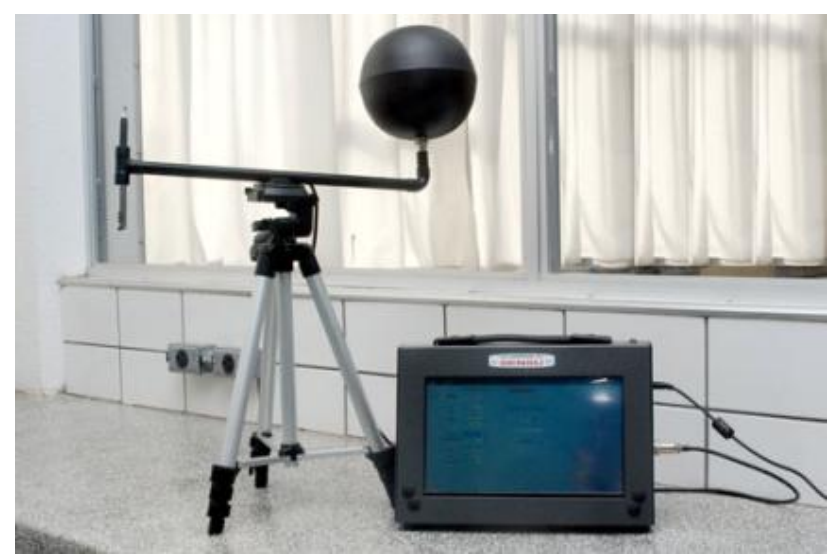


Figura 2 - Termoanemômetro portátil

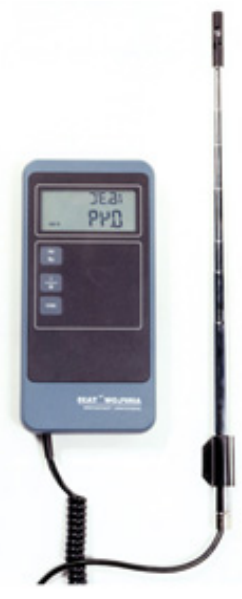

Os experimentos ocorreram nos turnos matutino e vespertino. Ao chegar às edificações, os pesquisadores instalavam as duas estações microclimáticas nos ambientes. Após a instalação dos equipamentos, era feita uma varredura na sala e desenhado um croqui especificando as dimensões do ambiente, posicionamento das estações de trabalho, localização de janelas, posição dos aparelhos de ar-condicionado e divisórias. Além disso, ainda era feita a medição pontual com o termoanemômetro, dos locais onde apareciam correntes de ar e realizavam-se anotações sobre as variações provocadas pelos ocupantes, como, por exemplo: abertura e fechamento de janelas, ajuste do sistema de ar-condicionado, alteração de vestimenta, etc.

\section{Tratamento dos dados}

A planilha de dados utilizada neste trabalho, com as respostas subjetivas dos usuários associadas às variáveis ambientais, corresponde à utilizada por Vecchi (2015) em sua tese de doutorado. Para a análise dos dados, primeiramente realizou-se uma análise descritiva, apresentando a distribuição de valores de acordo com a frequência de ocorrência e a variabilidade, e valores de máximo, mínimo, média e desvio padrão. Logo após, realizaram-se testes de hipóteses (qui-quadrado) e testes não paramétricos (correlação de Spearman) entre as variáveis a fim de verificar como estas se influenciam. Por meio dos testes foi possível verificar se as variáveis apresentam, estatisticamente, relação significativa. Compararam-se os valores resultantes do tratamento de dados com a conhecida classificação do IMC $^{1}$ (Tabela 1) e frequência de atividades físicas com as variáveis de sensação térmica, preferência térmica, conforto térmico, aceitabilidade térmica e valores de isolamento de roupas (clo).

As variáveis podem estar relacionadas (Tabela 2) de forma direta - quando o coeficiente $r$ de Spearman estiver no intervalo $(0<\mathrm{r}<1)$ - e de forma inversa $(-1<\mathrm{r}<0)$. Ou seja, para o caso de as variáveis estarem relacionadas de forma inversa, tem-se uma variável aumentando, enquanto a outra diminui.

\section{Resultados}

A análise de dados baseou-se em 85 estudos de campo realizados durante o ano de 2014 em diferentes estações climáticas. Essa pesquisa contou com a participação de 584 pessoas das três edificações, totalizando 2.695 votos de conforto térmico (Tabela 3). Destes, 1.199 foram votos femininos $(44,5 \%$ do total) e 1.496 votos masculinos (55,5\%). Obtiveram-se 1.620 votos em ambientes com aparelhos de ar-condicionado ligados (60,1\%) e 1.055 votos em ambientes ventilados naturalmente (39,2\%). Quanto ao tipo de edificação, obtiveram-se 1.275 votos em edificação com ar-condicionado central (edificação A), isto é, $47,3 \%$ do total de votos e 1.420 votos em edificações com ventilação híbrida (52,7\% do total de respostas - edificações B e C).

Ao se observarem os valores das características ambientais (Tabela 4), separados de acordo com os modos de operação, percebe-se que na edificação com ar-condicionado central a temperatura se manteve mais constante no ambiente, com mínima muito próxima da temperatura máxima. Quanto à umidade relativa do ar, percebe-se que o ar se torna mais seco devido à presença do ar-condicionado ligado.

${ }^{1}$ Calculou-se o IMC por meio da divisão da massa pela altura ao quadrado. 


\section{Vestimentas}

Em ambos os modos de operação há uma tendência dos valores de isolamento de roupas diminuírem conforme se aumentam os valores de IMC (Figura 3). Ao se comparar a frequência de atividades físicas com o isolamento de roupas, independentemente do modo de operação, não se obteve associação entre as variáveis.

Os resultados dos testes de hipóteses realizados para verificar a relação existente entre o isolamento de roupas com o IMC e a frequência de atividades físicas indicaram que a correlação entre frequência de atividades físicas e isolamento de roupas não foi significativa para ambos os modos de operação (Tabela 5). No entanto, ao se analisar o IMC percebe-se que houve correlação muito fraca, porém significativa, apenas na edificação que opera com ar-condicionado central, de modo que quanto maiores os valores de IMC, menores foram os valores de isolamento de roupas - ou seja, apesar de muito fraca a relação entre as variáveis, estatisticamente elas estão relacionadas.

Tabela 1 - Relação entre IMC e grupos de risco

\begin{tabular}{l|c|c|c|c|c|c}
\hline IMC $\left(\mathbf{k g} / \mathbf{m}^{2}\right)$ & $<\mathbf{1 8 , 5}$ & $\mathbf{1 8 , 5 - 2 4 , 9}$ & $\mathbf{2 5 , 0 - 2 9 , 9}$ & $\mathbf{3 0 , 0 - 3 4 , 9}$ & $\mathbf{3 5 , 0 - 3 9 , 9}$ & $>\mathbf{4 0 , 0}$ \\
\hline Situação & Abaixo do peso & Peso normal & $\begin{array}{c}\text { Acima do } \\
\text { peso }\end{array}$ & Obesidade I & $\begin{array}{c}\text { Obesidade II } \\
\text { (severa) }\end{array}$ & $\begin{array}{c}\text { Obesidade } \\
\text { III (mórbida) }\end{array}$ \\
\hline
\end{tabular}

Fonte: World Health Organization (2017).

Tabela 2 - Relação entre valores de $\mathbf{r}$ (em módulo) e tipo de relação

\begin{tabular}{c|c|c|c|c|c}
\hline Valor de $\mathbf{r}$ & $\mathbf{0 , 0 0}$ a $\mathbf{0 , 2 5}$ & $\mathbf{0 , 2 5}$ a 0,50 & $\mathbf{0 , 5 0}$ a $\mathbf{0 , 7 5}$ & $\mathbf{0 , 7 5}$ a $\mathbf{0 , 9 0}$ & $\mathbf{0 , 9 0}$ a $\mathbf{1 , 0 0}$ \\
\hline Tipo de relação & Muito fraca & Fraca & Moderada & Forte & Muito Forte \\
\hline
\end{tabular}

Fonte: Finney (1980).

Tabela 3 - Descrição estatística das características físicas dos indivíduos

\begin{tabular}{l|c|c|c|c|c|c|c|c}
\hline \multicolumn{1}{c|}{ Variáveis } & \multicolumn{2}{c|}{$\begin{array}{c}\text { Atividade } \\
\text { metabólica }\left(\mathbf{W} / \mathbf{m}^{2}\right)\end{array}$} & \multicolumn{2}{c|}{ Altura (m) } & \multicolumn{2}{c|}{ Peso (kg) } & \multicolumn{2}{c}{$\begin{array}{c}\text { Vestimenta } \\
\text { (clo) }\end{array}$} \\
\hline Tipo de edificação & Eac & Evh & Eac & Evh & Eac & Evh & Eac & Evh \\
Número de observações (N) & 1275 & 1420 & 1275 & 1420 & 1275 & 1420 & 1275 & 1420 \\
Mínimo & 1,00 & 1,00 & 1,50 & 1,50 & 42 & 43 & 0,42 & 0,31 \\
Máximo & 1,40 & 1,40 & 1,97 & 1,92 & 108 & 130 & 1,40 & 1,49 \\
Média & 1,04 & 1,02 & 1,72 & 1,70 & 75 & 74 & 0,68 & 0,72 \\
Desvio padrão & $\pm 0,10$ & $\pm 0,10$ & $\pm 0,10$ & $\pm 0,10$ & \pm 14 & \pm 16 & $\pm 0,20$ & $\pm 0,20$ \\
\hline
\end{tabular}

Nota: Eac indica edificação com sistema de ar-condicionado central e Evh indica edificações com ventilação híbrida.

Tabela 4 - Descrição estatística das características ambientais

\begin{tabular}{l|c|c|c|c|c|c|c|c}
\hline \multicolumn{1}{c|}{ Variáveis } & \multicolumn{2}{|c|}{$\begin{array}{c}\text { Temperatura } \\
\text { interna }\left({ }^{\circ} \mathbf{C}\right)\end{array}$} & \multicolumn{2}{c|}{\begin{tabular}{c}
\multicolumn{2}{c|}{ Umidade relativa } \\
do ar (\%)
\end{tabular}} & \multicolumn{2}{c|}{$\begin{array}{c}\text { Temperatura } \\
\text { externa }\left({ }^{\circ} \mathbf{C}\right)\end{array}$} & \multicolumn{2}{c}{$\begin{array}{c}\text { Umidade } \\
\text { relativa } \\
\text { externa (\%) }\end{array}$} \\
\hline $\begin{array}{l}\text { Tipo de edificação } \\
\text { Número de }\end{array}$ & Eac & Evh & Eac & Evh & Eac & Evh & Eac & Evh \\
observações (N) & 1275 & 1420 & 1275 & 1420 & 1275 & 1420 & 1275 & 1420 \\
Mínimo & 21,50 & 17,10 & 23,05 & 38,70 & 12,20 & 12,20 & 59,00 & 59,00 \\
Máximo & 24,66 & 28,00 & 78,14 & 82,25 & 25,30 & 24,70 & 89,00 & 83,00 \\
Média & 23,01 & 23,19 & 60,95 & 62,08 & 20,03 & 19,37 & 72,00 & 71,00 \\
Desvio padrão & $\pm 0,56$ & $\pm 1,16$ & $\pm 9,29$ & $\pm 9,12$ & $\pm 4,31$ & $\pm 2,89$ & $\pm 6,99$ & $\pm 7,28$ \\
\hline
\end{tabular}

Nota: Eac indica edificação com sistema de ar-condicionado central e Evh indica edificações com ventilação híbrida. 
Figura 3 - Relação entre IMC e isolamento de roupas para os diferentes modos de operação

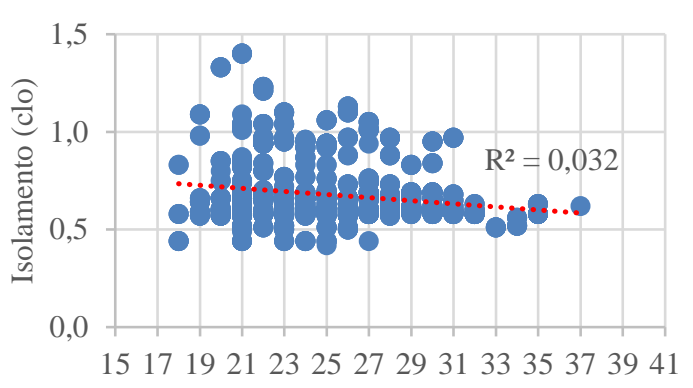

IMC

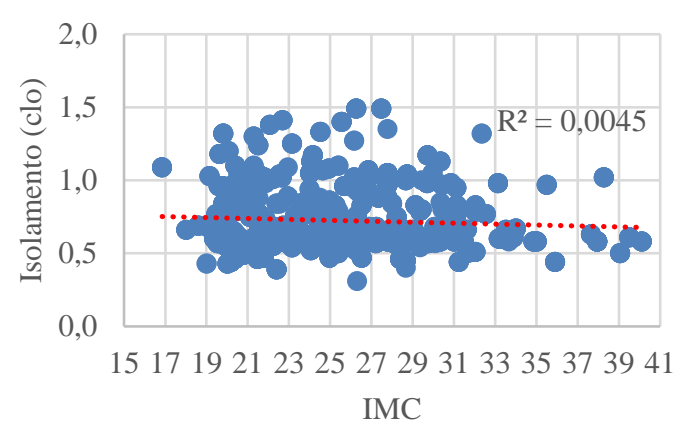

(b) Ventilação híbrida ( $\mathrm{N}=1420)$

(a) Ar-condicionado central $(\mathrm{N}=1275)$

Tabela 5 - Resultados obtidos nos testes de hipóteses para isolamento de roupas

\begin{tabular}{l|c|c|c|c}
\hline \multirow{2}{*}{ Testes de hipóteses } & \multicolumn{2}{|c|}{ Ar-condicionado central } & \multicolumn{2}{c}{ Ventilação híbrida } \\
\cline { 2 - 5 } & $\begin{array}{c}\text { Índice de massa } \\
\text { corpórea }\end{array}$ & $\begin{array}{c}\text { Frequência de } \\
\text { atividades físicas }\end{array}$ & $\begin{array}{c}\text { Índice de massa } \\
\text { corpórea }\end{array}$ & $\begin{array}{c}\text { Frequência de } \\
\text { atividades físicas }\end{array}$ \\
\hline $\begin{array}{l}\text { Valor de p (qui- } \\
\text { quadrado) }\end{array}$ & 0,00001 & 0,00001 & 0,00001 & 0,00001 \\
\hline Valor de p (Spearman) & 0,00224 & 0,46628 & 0,08290 & 0,21659 \\
\hline $\begin{array}{l}\text { Coeficiente r de } \\
\text { Spearman }\end{array}$ & $-0,08002$ & 0,00239 & 0,04983 & 0,02819 \\
\hline
\end{tabular}

\section{Sensação térmica}

A sensação térmica é medida pela escala da ASHRAE e indica como o usuário está se sentindo termicamente, variando de -3 (muito frio) a +3 (muito calor). Analisando-se as porcentagens de votos de sensação térmica obtidas com relação às faixas de IMC e em função do modo de operação (Figura 4), percebe-se que na edificação que opera com ar-condicionado central não houve votos na categoria "Muito Calor (+3)". Observa-se que os votos para sensação térmica neutra (0) se mostraram superiores para ambientes com ar-condicionado central. Em ambos os modos, nota-se diminuição na sensação de frio conforme se aumentam os valores de IMC, com exceção do IMC “Obesidade I” no modo de ventilação híbrida. Assim, percebe-se uma tendência de que indivíduos com maior IMC sentem o ambiente mais aquecido.

Por meio da análise da porcentagem dos votos de sensação térmica e da frequência de atividades físicas para os dois modos de operação (Figura 5), observou-seque os valores para sensação térmica neutra aumentaram em direção ao sedentarismo (indivíduos que nunca praticam exercícios físicos) apenas na edificação que opera com arcondicionado central. Nas edificações com ventilação híbrida houve aumento nos votos para "pouco frio (-1)" e diminuição dos votos para "pouco calor $(+1)$ " à medida que se aumenta a prática de exercícios físicos.
Os resultados obtidos nos testes estatísticos para sensação térmica (Tabela 6) indicaram que para ambos os modos de operação houve correlação significativa entre IMC e sensação térmica. Porém, a correlação foi muito fraca para as edificações que operaram com ventilação híbrida e para a edificação com ar-condicionado central. Logo, conforme se aumentam os valores de IMC, há tendência de os indivíduos sentirem-se mais aquecidos, independentemente do modo de operação. Esse resultado é semelhante aos encontrados na literatura (LEITES et al., 2013; FADEYI, 2014). Para a frequência de atividades pode-se concluir que a correlação entre essa variável e a sensação térmica não foi significativa para a edificação que operou com ar-condicionado central. No entanto, para as edificações com ventilação híbrida, confirmou-se que a correlação entre as variáveis, apesar de muito fraca, foi significativa, ou seja, apesar de muito fraca a relação entre as variáveis, estatisticamente elas estão relacionadas. Assim, indivíduos que se exercitam regularmente sentem o ambiente térmico mais resfriado.

\section{Preferência térmica}

A porcentagem de votos de preferência térmica que os indivíduos de diferentes IMCs apresentaram para cada modo de operação (Figura 6) indicou que há diminuição nos votos de preferência "mais aquecidos", conforme se aumentam os valores de 
IMC para ar-condicionado central. Para ambientes com ventilação híbrida observou-se o contrário. As porcentagens de preferência térmica por não mudar (“assim mesmo”) foram maiores em usuários obesos e acima do peso na edificação com sistema de ar-condicionado central e nos ambientes com ventilação híbrida, respectivamente.

\section{Figura 4 - Relação entre IMC e sensação térmica para os diferentes modos de operação}

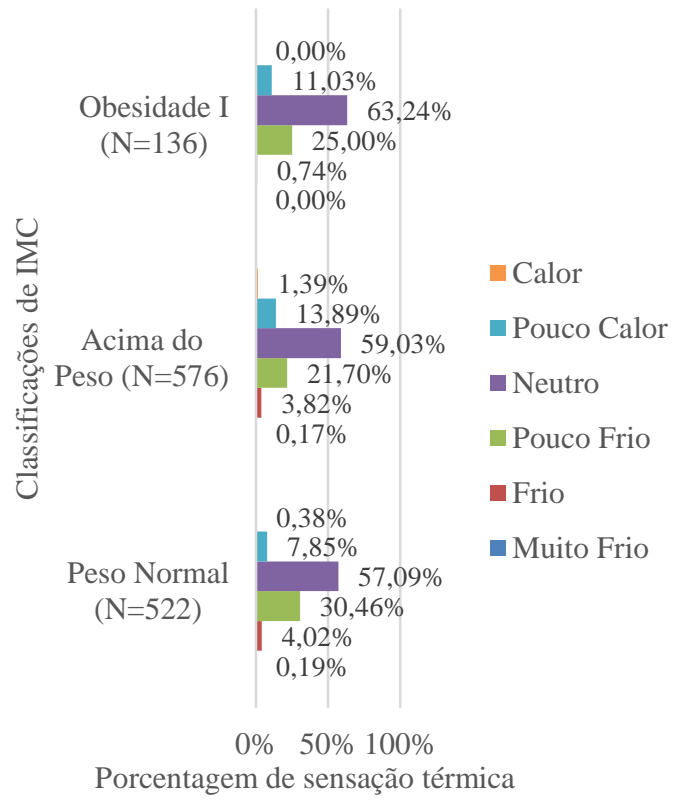

(a) Ar-condicionado central

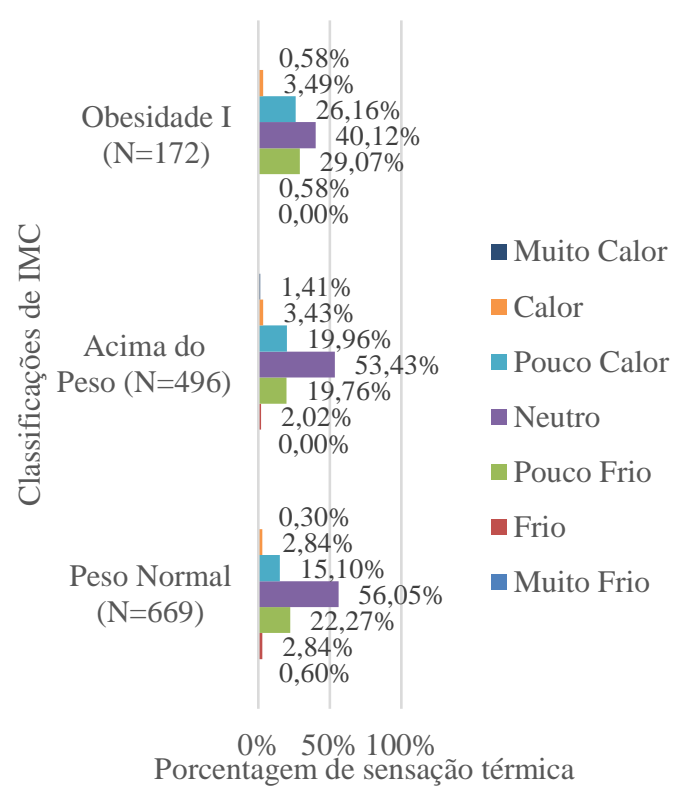

(b) Ventilação híbrida

\section{Figura 5 - Relação entre frequência de atividades físicas e sensação térmica para os diferentes modos de operação}

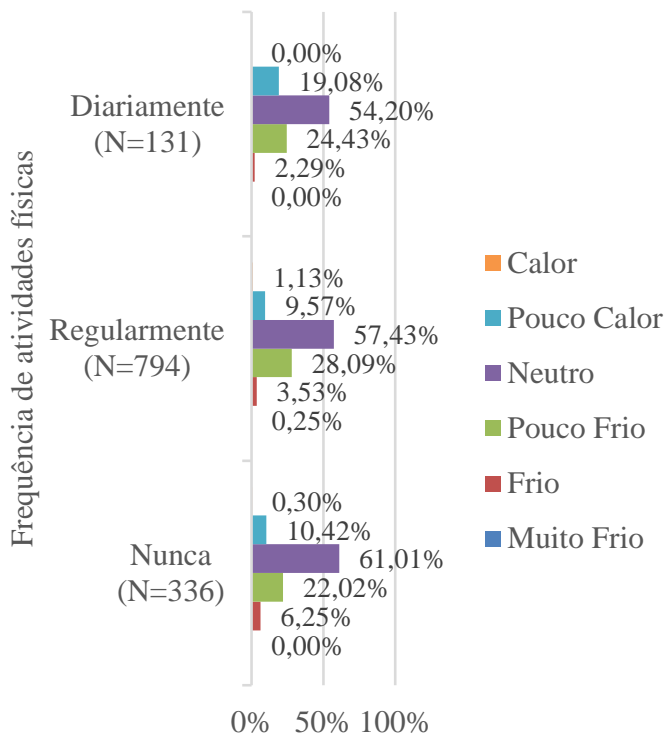

Porcentagem de sensação térmica

(a) Ar-condicionado central

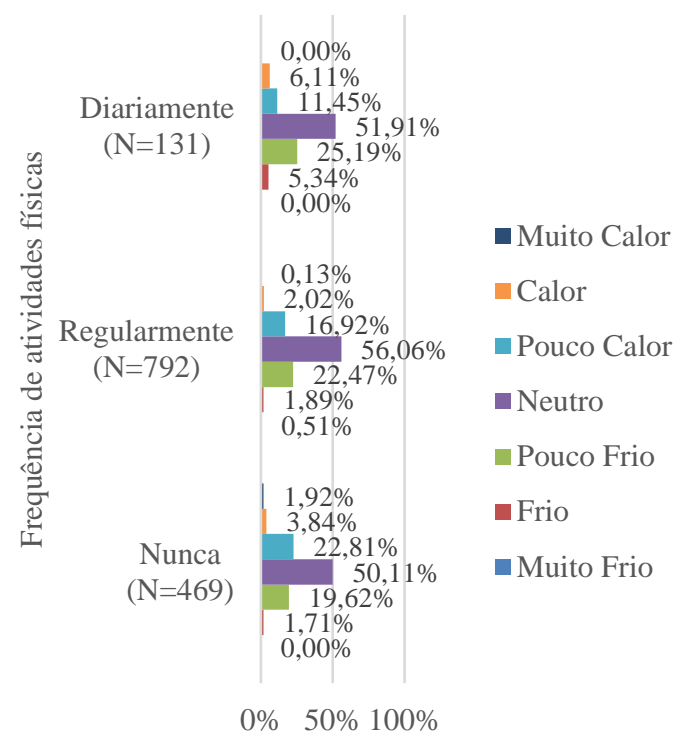

Porcentagem de sensação térmica

(b) Ventilação híbrida 
Tabela 6 - Resultados obtidos nos testes de hipóteses para sensação térmica

\begin{tabular}{l|c|c|c|c}
\hline \multirow{2}{*}{ Testes de hipóteses } & \multicolumn{2}{|c|}{ Ar-condicionado central } & \multicolumn{2}{c}{ Ventilação híbrida } \\
\cline { 2 - 5 } & $\begin{array}{c}\text { Índice de massa } \\
\text { corpórea }\end{array}$ & $\begin{array}{c}\text { Frequência de } \\
\text { atividades físicas }\end{array}$ & $\begin{array}{c}\text { Índice de massa } \\
\text { corpórea }\end{array}$ & $\begin{array}{c}\text { Frequência de } \\
\text { atividades físicas }\end{array}$ \\
\hline $\begin{array}{l}\text { Valor de p (qui- } \\
\text { quadrado) }\end{array}$ & 0,00001 & 0,00001 & 0,00001 & 0,00001 \\
\hline Valor de p (Spearman) & 0,00001 & 0,22428 & 0,00001 & 0,00003 \\
\hline $\begin{array}{l}\text { Coeficiente r de } \\
\text { Spearman }\end{array}$ & 0,13619 & $-0,02136$ & 0,24343 & 0,12145 \\
\hline
\end{tabular}

Figura 6 - Relação entre os valores de IMC e preferência térmica

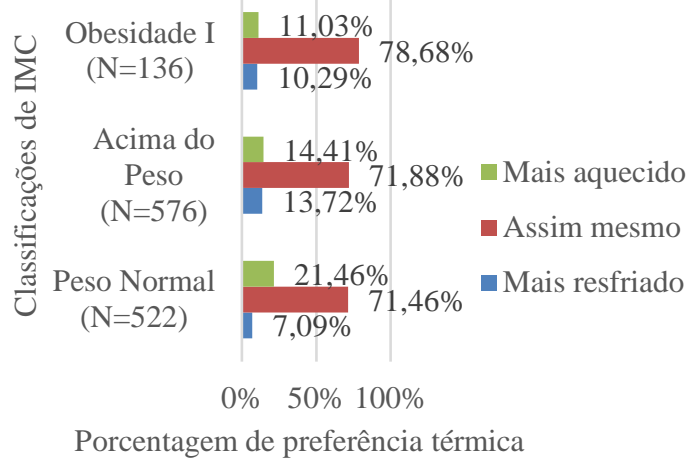

(a) Ar-condicionado central

Para os votos de preferência térmica relacionados com a frequência de atividades físicas, nota-se que em ambientes com ar-condicionado central há aumento na preferência por ambientes mais aquecidos conforme se diminui a frequência de atividades físicas, bem como diminuição na preferência por não mudar os ambientes (Figura 7). Ao se analisar os valores de preferência em ambientes com ventilação híbrida, nota-se que há maior preferência por ambientes mais resfriados conforme se aumenta a frequência de atividades físicas.

Por meio dos dados obtidos nos testes de hipóteses, indicados na Tabela 7 , concluiu-se que há correlação significativa entre as variáveis de preferência térmica e IMC para ambos os modos de operação. Ao se observar o coeficiente $r$ de Spearman, percebe-se que ambas as correlações foram muito fracas. Conforme se aumentam os valores de IMC, há maior preferência por ambientes mais resfriados. Para frequência de atividades físicas, somente a edificação que opera com arcondicionado central mostrou correlação que, apesar de muito fraca, foi significativa. Assim, percebe-se que indivíduos que praticam atividades físicas regularmente preferem um ambiente térmico mais resfriado do que aqueles sedentários.

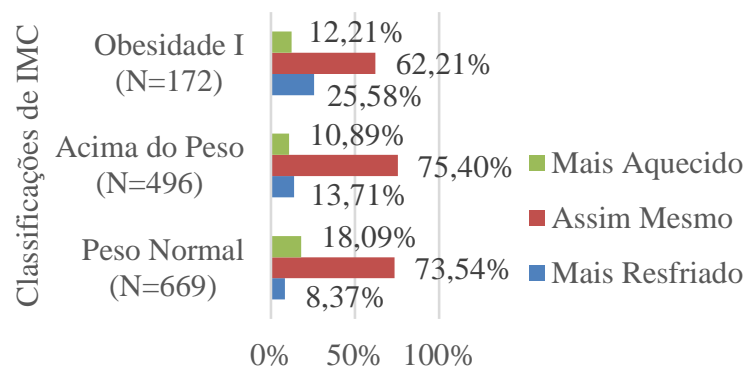

Porcentagem de preferência térmica

(b) Ventilação híbrida

\section{Conforto térmico}

A porcentagem de votos de conforto térmico para cada subgrupo de IMC separados por modo de operação (Figura 8) indicou que, de modo geral, a maior parte das pessoas estava se considerando confortável termicamente. No entanto, para a edificação que operou com ar-condicionado central há certa linearidade no crescimento dos votos para ambiente confortável à medida que os valores de IMC aumentam. Porém, o mesmo não pode ser dito para as edificações que operaram com ventilação híbrida, pois há maior porcentagem de indivíduos confortáveis para o grupo considerado acima do peso do que para o grupo de obesos.

Por meio da análise da porcentagem de votos de conforto térmico para cada subgrupo de frequência de atividades físicas (Figura 9), notou-se que na edificação que operou com ar-condicionado central os votos mostraram-se maiores para desconfortável termicamente, crescendo conforme a menor frequência de exercícios durante a semana (opções "nunca” e “semanalmente”). Enquanto nas edificações que operaram com ventilação híbrida, os votos para maior desconforto térmico foram para indivíduos que se exercitam diariamente. Em ambas as edificações os votos indicaram alta porcentagem de conforto térmico (acima de 90\%). 
Figura 7 - Relação entre os valores de frequência de atividades físicas e preferência térmica

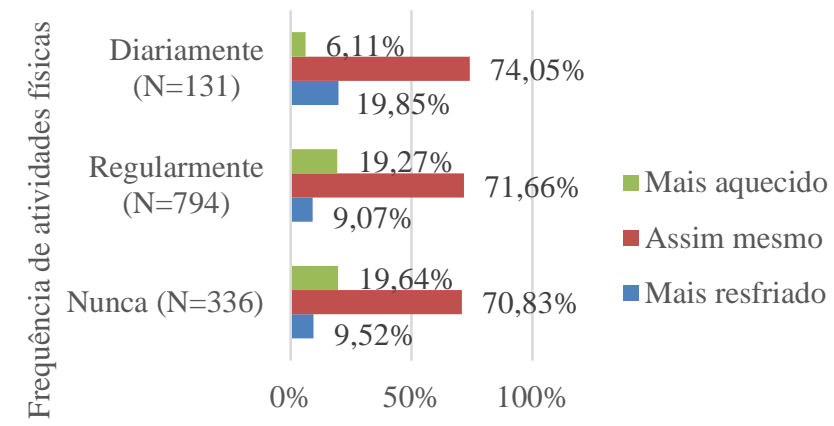

Porcentagem de preferência térmica

(a) Ar-condicionado central

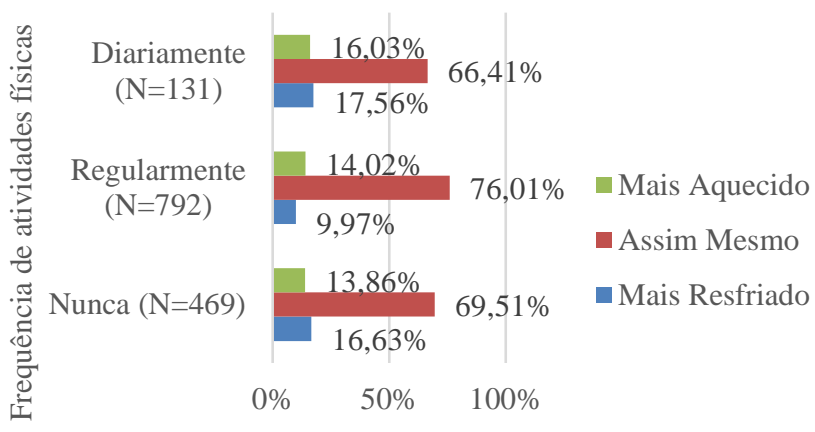

Porcentagem de preferência térmica

(b) Ventilação híbrida

Tabela 7 - Resultados obtidos nos testes de hipóteses para preferência térmica

\begin{tabular}{l|c|c|c|c}
\hline \multirow{2}{*}{ Testes de hipóteses } & \multicolumn{2}{|c|}{ Ar-condicionado central } & \multicolumn{2}{c}{ Ventilação híbrida } \\
\cline { 2 - 5 } & $\begin{array}{c}\text { Índice de massa } \\
\text { corpórea }\end{array}$ & $\begin{array}{c}\text { Frequência de } \\
\text { atividades físicas }\end{array}$ & $\begin{array}{c}\text { Índice de massa } \\
\text { corpórea }\end{array}$ & $\begin{array}{c}\text { Frequência de } \\
\text { atividades físicas }\end{array}$ \\
\hline $\begin{array}{l}\text { Valor de p (qui- } \\
\text { quadrado) }\end{array}$ & 0,00001 & 0,00001 & 0,00001 & 0,00001 \\
\hline Valor de p (Spearman) & 0,00001 & 0,00127 & 0,00001 & 0,27567 \\
\hline $\begin{array}{l}\text { Coeficiente r de } \\
\text { Spearman }\end{array}$ & 0,16941 & $-0,08497$ & 0,19938 & 0,01594 \\
\hline
\end{tabular}

Figura 8 - Relação entre os valores de IMC e conforto térmico

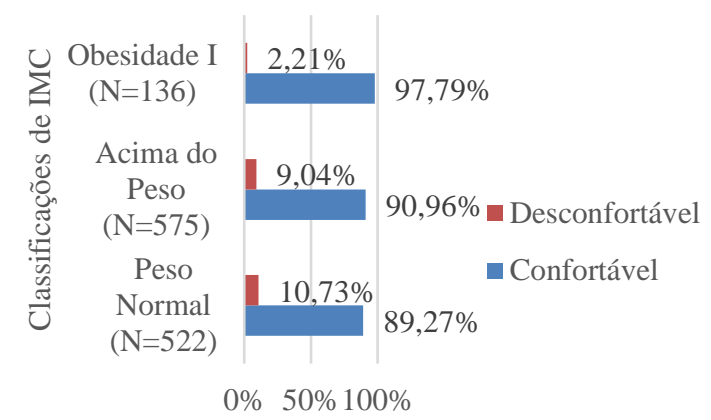

Porcentagem de conforto térmico

(a) Ar-condicionado central

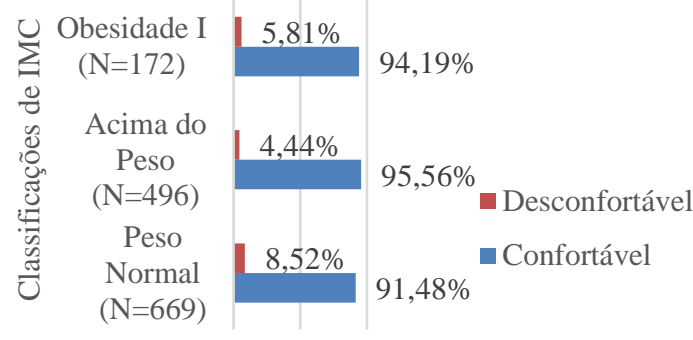

0\% 50\% $100 \%$

Porcentagem de conforto térmico

(b) Ventilação híbrida 


\section{Figura 9 - Relação entre os valores de frequência de atividades físicas e conforto térmico}

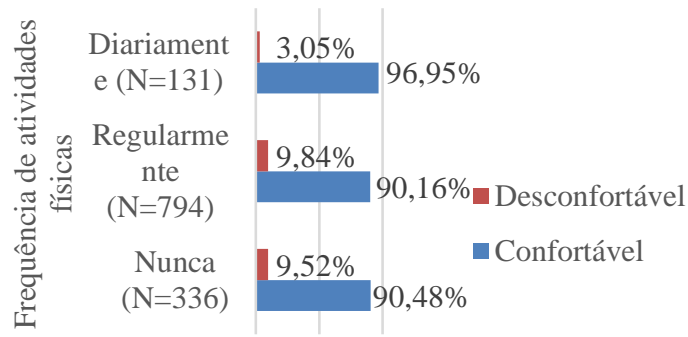

$0 \% 50 \% 100 \%$

Porcentagem de conforto térmico

(a) Ar-condicionado central

Ao se analisar os valores resultantes dos testes de hipóteses, nota-se que o IMC e o conforto térmico apresentaram correlação significativa, apesar de muito fraca (Tabela 8) para ambos os modos de operação. Pelo valor do coeficiente r de Spearman, concluiu-se que essa correlação se mostrou de forma inversa, ou seja, conforme se aumentam os valores de IMC, menor é o desconforto térmico. Para frequência de atividades físicas, percebe-se que houve correlação muito fraca, porém significativa, na edificação que operou com ventilação híbrida. $\mathrm{Na}$ edificação com arcondicionado central não houve correlação significativa entre frequência de atividades físicas e conforto térmico.

\section{Aceitabilidade térmica}

Analisando-se a porcentagem de votos de aceitabilidade térmica, separados conforme cada subgrupo de IMC e pelo modo de operação (Figura 10), percebeu-se que para a edificação com arcondicionado central os votos para ambiente aceitável foram muito próximos nas classificações de IMC “peso normal” e "acima do peso”, enquanto para indivíduos obesos houve $100 \%$ dos votos para ambiente térmico aceitável. Para ventilação híbrida, novamente houve predominância de votos para ambiente aceitável, sendo levemente superiores quando comparados com a edificação que opera com ar-condicionado central, exceto para indivíduos obesos. Tal grupo apresentou redução nos votos de aceitabilidade térmica.

Ao se analisar a relação entre a frequência de atividades físicas e a aceitabilidade térmica, apresentada na Figura 11, nota-se que para ambos os modos de operação indivíduos que se exercitam diariamente apresentaram maiores valores de

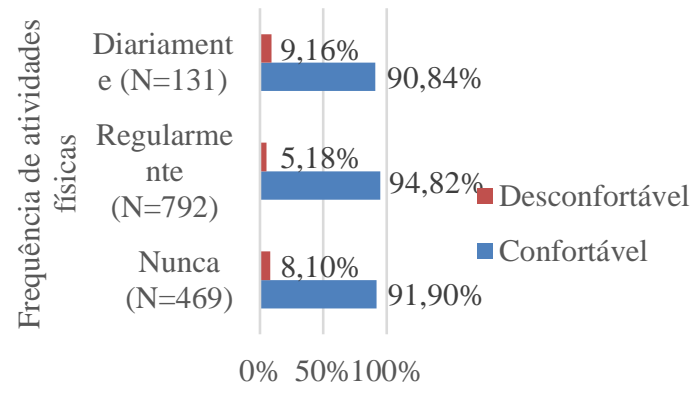

Porcentagem de conforto térmico

(b) Ventilação híbrida

aceitabilidade térmica. No entanto, para ambientes com ar-condicionado central, os votos de aceitabilidade térmica se mostraram muito próximos entre indivíduos que se exercitam regularmente e aqueles que não praticam atividade física. Porém, analisando os ambientes com ventilação híbrida, indivíduos que se exercitam regularmente aceitam melhor o ambiente do que aqueles que não o fazem. Porém, os valores percentuais ficaram muito próximos entre ambos os subgrupos.

Os resultados obtidos por meio da aplicação dos testes de hipóteses (Tabela 9) indicaram que houve correlação significativa, apesar de muito fraca, entre IMC e aceitabilidade térmica apenas na edificação com ar-condicionado central, de forma que indivíduos com maiores valores de IMC aceitam melhor o ambiente do que aqueles com menores valores. Para frequência de atividades físicas, houve correlação significativa, apesar de muito fraca, apenas para as edificações que operam com ventilação híbrida, de forma que conforme se aumenta a frequência de atividades físicas durante a semana, maiores são os índices de aceitabilidade térmica.

\section{Síntese dos resultados}

Para a edificação A, com sistema de arcondicionado central, todos os valores de IMC mostraram associação com a vestimenta e os indicadores de conforto (Tabela 10). Vestimenta e conforto térmico associaram-se inversamente, enquanto sensação, preferência e aceitabilidade térmica associaram-se diretamente. No entanto, observou-se que a preferência térmica foi a única variável que se associou com a frequência de atividades físicas. 
Tabela 8 - Resultados obtidos nos testes de hipóteses para conforto térmico

\begin{tabular}{l|c|c|c|c}
\hline \multirow{2}{*}{ Testes de hipóteses } & \multicolumn{2}{|c|}{ Ar-condicionado central } & \multicolumn{2}{c}{ Ventilação híbrida } \\
\cline { 2 - 5 } & $\begin{array}{c}\text { Índice de massa } \\
\text { corpórea }\end{array}$ & $\begin{array}{c}\text { Frequência de } \\
\text { atividades físicas }\end{array}$ & $\begin{array}{c}\text { Índice de massa } \\
\text { corpórea }\end{array}$ & $\begin{array}{c}\text { Frequência de } \\
\text { atividades físicas }\end{array}$ \\
\hline $\begin{array}{l}\text { Valor de p (qui- } \\
\text { quadrado) }\end{array}$ & 0,00001 & 0,00001 & 0,00001 & 0,00001 \\
\hline Valor de p (Spearman) & 0,00001 & 0,07631 & 0,00001 & 0,02049 \\
\hline $\begin{array}{l}\text { Coeficiente r de } \\
\text { Spearman }\end{array}$ & $-0,12869$ & 0,04032 & $-0,09428$ & $-0,02205$ \\
\hline
\end{tabular}

Figura 10 - Relação entre os valores de IMC e aceitabilidade térmica

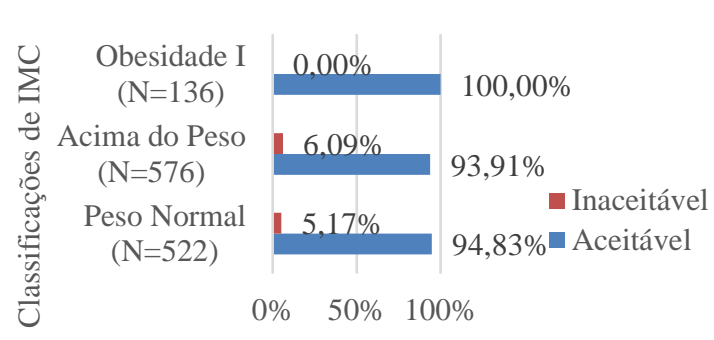

Porcentagem de aceitabilidade térmica

(a) Ar-condicionado central

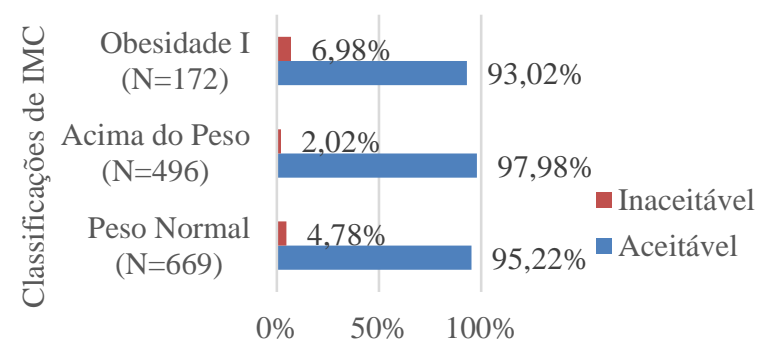

Porcentagem de aceitabilidade térmica

(b) Ventilação híbrida

Figura 11 - Relação entre os valores de frequência de atividades físicas e aceitabilidade térmica

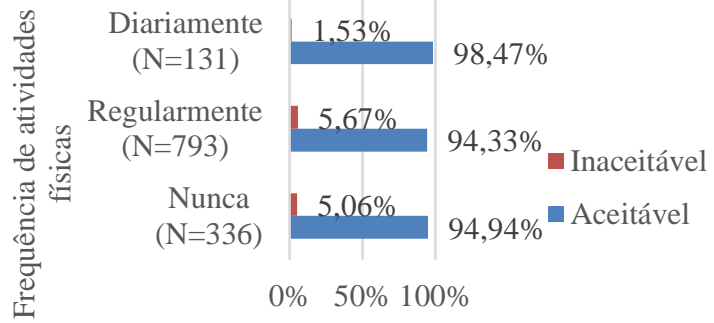

Porcentagem de aceitabilidade térmica

(a) Ar-condicionado central

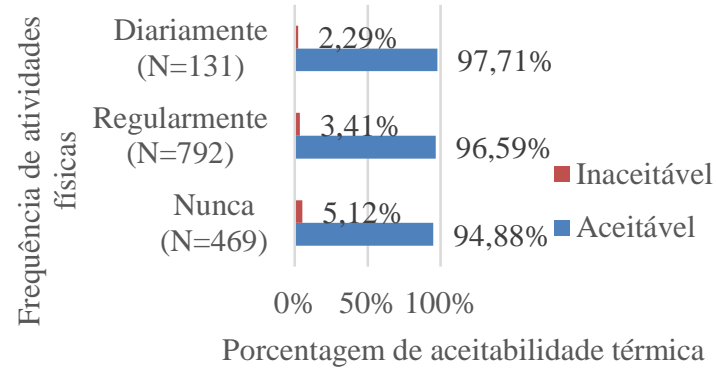

(b) Ventilação híbrida

Tabela 9 - Resultados obtidos nos testes de hipóteses para aceitabilidade térmica

\begin{tabular}{l|c|c|c|c}
\hline \multirow{2}{*}{ Testes de hipóteses } & \multicolumn{2}{|c|}{ Ar-condicionado central } & \multicolumn{2}{c}{ Ventilação híbrida } \\
\cline { 2 - 5 } & $\begin{array}{c}\text { Índice de massa } \\
\text { corpórea }\end{array}$ & $\begin{array}{c}\text { Frequência de } \\
\text { atividades físicas }\end{array}$ & $\begin{array}{c}\text { Índice de massa } \\
\text { corpórea }\end{array}$ & $\begin{array}{c}\text { Frequência de } \\
\text { atividades físicas }\end{array}$ \\
\hline $\begin{array}{l}\text { Valor de p (qui- } \\
\text { quadrado) }\end{array}$ & 0,00001 & 0,00001 & 0,00001 & 0,00001 \\
\hline Valor de p (Spearman) & 0,00091 & 0,19186 & 0,11527 & 0,01894 \\
\hline $\begin{array}{l}\text { Coeficiente r de } \\
\text { Spearman }\end{array}$ & $-0,08772$ & 0,02455 & $-0,03208$ & 0,05552 \\
\hline
\end{tabular}


Tabela 10 - Síntese dos resultados obtidos na edificação com sistema de ar-condicionado central

\begin{tabular}{l|c|c}
\hline \multicolumn{1}{c|}{ Variáveis } & IMC & Frequência de atividades físicas \\
\hline Vestimenta (clo) & Associação (Inversa) & Sem associação \\
Sensação térmica & Associação (Direta) & Sem associação \\
Conforto térmico & Associação (Inversa) & Sem associação \\
Preferência térmica & Associação (Direta) & Associação (Inversa) \\
Aceitabilidade térmica & Associação (Direta) & Sem associação \\
\hline
\end{tabular}

Para as edificações B e C, com sistema híbrido, a vestimenta não mostrou associação com nenhuma variável (Tabela 11). A preferência térmica apresentou associação direta quando relacionada com os valores de IMC nas duas edificações; enquanto os outros indicadores mostraram-se diferentes em cada edificação. Considerando a frequência de atividades físicas, nota-se que para a edificação B houve associação somente com a variável de conforto térmico, enquanto na edificação C somente associou-se com sensação térmica, ambas de forma direta.

Também se realizaram análises separadas de cada classificação de IMC e comparações com as mesmas variáveis. Indivíduos com peso normal apresentaram associação de forma direta com a preferência térmica nas três edificações. A aceitabilidade térmica se relacionou com os indivíduos com peso normal apenas nas edificações com ventilação híbrida, enquanto na edificação que opera com ar-condicionado central não houve associação.

Indivíduos acima do peso também apresentaram associação direta com a preferência térmica nas três edificações. No entanto, a vestimenta associou-se apenas com a edificação que opera com arcondicionado central. Sensação, conforto e aceitabilidade térmica associaram-se diretamente apenas nas edificações que operam com sistema de ventilação híbrida.

A obtenção desses resultados, mostrando as diferentes percepções térmicas dos trabalhadores de escritórios de acordo com seu $\mathrm{IMC}^{2}$, ressalta a necessidade de rever os conceitos sobre os requisitos de homogeneização térmica dos ambientes, conforme prescrita pela teoria de conforto analítico de Fanger (1970). Ambos os tipos de edificação (com sistema central de arcondicionado e com ventilação híbrida) apresentaram resultados similares de conforto térmico, ou seja, os usuários das edificações com ventilação híbrida toleraram maior variação das condições térmicas internas e utilizaram a ventilação natural de acordo com as condições

${ }^{2}$ Diferenças no conforto térmico entre usuários do sexo masculino e feminino também se observaram em edificações de escritório em Florianópolis (MAYKOT; RUPP; GHISI, 2018). climáticas externas. Isso vai ao encontro da teoria de conforto térmico adaptativo (DE DEAR; BRAGER, 1998; NICOL; HUMPHREYS, 2002). Assim, considerando um ano inteiro, as edificações com ventilação híbrida tendem a consumir menos energia com o sistema de ar-condicionado do que a edificação com sistema central de ar-condicionado.

Nas edificações estudadas, com o intuito de considerar as diferenças individuais no conforto térmico, poderiam ser empregados sistemas de condicionamento personalizado (VESELÝ; ZEILER, 2014; ZHANG; ARENS; ZHAI, 2015). Dessa maneira, os usuários poderiam ajustar as condições térmicas de seus respectivos entornos imediatos de acordo com suas preferências.

\section{Conclusões}

Neste trabalho, por se tratar de estudos realizados em edificações, com pessoas desempenhando suas atividades corriqueiras, e não em câmaras climáticas, as análises consideraram o índice de massa corpórea (IMC), que relaciona a massa corporal e a altura, como parâmetro de classificação de obesos, pessoas com peso normal e pessoas abaixo do peso. Porém, cabe salientar que o IMC não mede a gordura corporal e nem reflete adequadamente a proporção entre músculo e gordura, o que pode levar a classificações errôneas (AHIMA; LAZAR, 2013).

Todas as conclusões aqui apresentadas são referentes a estudos de campo envolvendo a aplicação de questionários ao mesmo tempo em que as variáveis térmicas eram medidas. Os estudos foram realizados durante o ano de 2014 em diferentes estações climáticas, em três edificações de escritórios localizadas no clima subtropical da cidade de Florianópolis, SC. Duas das edificações operaram com ventilação híbrida e a outra edificação possuía sistema de ar-condicionado central. Com base nos resultados apresentados é possível concluir que em ambos os modos de operação há tendência de o isolamento de roupas diminuir conforme se aumentam os valores de IMC.

Diferentes grupos de pessoas possuem diferentes requisitos térmicos. 
Tabela 11 - Síntese dos resultados obtidos nas edificações com sistema híbrido

\begin{tabular}{l|c|c|c|c|c}
\hline \multicolumn{2}{|c|}{ Edificação B } & \multicolumn{2}{c}{ Edificação C } \\
\hline Variáveis & IMC & $\begin{array}{c}\text { Frequência de } \\
\text { atividades físicas }\end{array}$ & Variáveis & IMC & $\begin{array}{c}\text { Frequência de } \\
\text { atividades físicas }\end{array}$ \\
\hline $\begin{array}{l}\text { Vestimenta } \\
\text { (clo) }\end{array}$ & $\begin{array}{c}\text { Sem } \\
\text { associação }\end{array}$ & Sem associação & $\begin{array}{c}\text { Vestimenta } \\
\text { (clo) }\end{array}$ & $\begin{array}{c}\text { Sem } \\
\text { associação }\end{array}$ & Sem associação \\
\hline $\begin{array}{l}\text { Sensação } \\
\text { térmica }\end{array}$ & $\begin{array}{c}\text { Sem } \\
\text { associação }\end{array}$ & Sem associação & $\begin{array}{c}\text { Sensação } \\
\text { térmica }\end{array}$ & $\begin{array}{c}\text { Associação } \\
\text { (Direta) }\end{array}$ & Associação (Direta) \\
\hline $\begin{array}{l}\text { Conforto } \\
\text { térmico }\end{array}$ & $\begin{array}{c}\text { Associação } \\
\text { (Inversa) }\end{array}$ & Associação (Direta) & $\begin{array}{c}\text { Conforto } \\
\text { térmico }\end{array}$ & $\begin{array}{c}\text { Sem } \\
\text { associação }\end{array}$ & Sem associação \\
\hline $\begin{array}{l}\text { Preferência } \\
\text { térmica }\end{array}$ & $\begin{array}{c}\text { Associação } \\
\text { (Direta) }\end{array}$ & Sem associação & $\begin{array}{c}\text { Preferência } \\
\text { térmica }\end{array}$ & $\begin{array}{c}\text { Associação } \\
\text { (Direta) }\end{array}$ & Sem associação \\
\hline $\begin{array}{l}\text { Aceitabilidad } \\
\text { e térmica }\end{array}$ & $\begin{array}{c}\text { Associação } \\
\text { (Direta) }\end{array}$ & Sem associação & $\begin{array}{c}\text { Aceitabilidad } \\
\text { e térmica }\end{array}$ & $\begin{array}{c}\text { Sem } \\
\text { associação }\end{array}$ & Sem associação \\
\hline
\end{tabular}

Em ambos os tipos de edificação constatou-se que há correlação significativa entre IMC e sensação térmica, preferência térmica e conforto térmico. Indivíduos com maior IMC sentem o ambiente mais aquecido e preferem ambientes mais resfriados esses resultados estão de acordo com os apresentados na literatura (LEITES et al., 2013; FADEYI, 2014). Usuários com maior IMC também se sentiram mais confortáveis termicamente. O IMC somente apresentou relação significativa com a aceitabilidade térmica na edificação com arcondicionado central, sendo que usuários com maiores valores de IMC aceitam em maior proporção o ambiente térmico. A influência do IMC na vestimenta e nas respostas subjetivas das pessoas provavelmente está relacionada com a adiposidade, pois impacta a transferência de calor entre o corpo e o ambiente. O aumento no percentual de conforto térmico com o aumento do IMC possivelmente é explicado pelo fato de os estudos de campo não terem sido realizados no verão. Assim, a maioria das reclamações de desconforto térmico reportadas pelos usuários deveu-se ao frio. Desse modo, o aumento do IMC diminuiu o desconforto térmico. Cabe salientar que essa constatação pode não ser verdadeira para as estações mais quentes.

Os resultados relacionados à frequência de atividades físicas não se mostraram conclusivos considerando todas as edificações. Em edificações com ventilação híbrida observou-se relação significativa entre frequência de atividades físicas e sensação, conforto e aceitabilidade térmica. Indivíduos que se exercitam regularmente sentiram o ambiente térmico mais resfriado, reportaram maior desconforto térmico, mas aceitaram em maior proporção o ambiente térmico do que usuários sedentários. Na edificação com sistema de arcondicionado central, indivíduos que praticam atividades físicas regularmente preferem um ambiente térmico mais resfriado do que aqueles sedentários.
Para melhor compreensão da influência de diferentes variáveis no conforto térmico, mais estudos e análises devem ser realizados considerando o verão em Florianópolis e também, de maneira geral, nas demais regiões do Brasil.

\section{Referências}

AHIMA, R. S.; LAZAR, M.A. The Health Risk of Obesity: better metrics imperative. Science, v. 341, n. 6148, p. 856-858, 2013.

\section{AMERICAN SOCIETY OF HEATING, REFRIGERATING AND AIR-CONDITIONING ENGINEERS. Standard 55: thermal environmental conditions for human occupancy. Atlanta, 2013.}

CLAESSENS-VAN OOIJEN, A. M. J. et al. Heat Production and Body Temperature During Cooling and Rewarming in Overweight and Lean Men. Obesity, v. 14, n. 11, p. 1.914-1.920, 2006.

DE DEAR, R. J.; BRAGER, G. Developing an Adaptive Model of Thermal Comfort and Preference. ASHRAE Transactions, v. 104, p. 145-167, 1998.

FADEYI, M. O. Initial Study on the Impact of Thermal History on Building Occupants' Thermal Assessments in Actual Air-Conditioned Office Buildings. Building and Environment, v. 80, p. 36-47, 2014.

FANGER, P. O. Thermal Comfort: analysis and applications in environmental engineering. Copenhagen: Danish Technical Press, 1970.

FINNEY, D.J. Statistics for Biologists. London: Chapman and Hall, 1980.

KINGMA, B.; FRIJNS, A.; VAN MARKEN LICHTENBELT, W. The Thermoneutral Zone: Implications for metabolic studies. Frontiers in Bioscience, v. 4E, n. 5, p. 1.975-1.985, 2012. 
LEITES, G. T. et al. Responses of Obese and Lean Girls Exercising under Heat and Thermoneutral Conditions. The Journal of Pediatrics, v. 162, n. 5, p. 1.054-60, 2013.

MAEDA, T. et al. Body Composition, and Physical Fitness on Cold Tolerance in Humans. Journal of Physiological Anthropology and Applied Human Science, v. 24, n. 4, p. 439-443, 2005.

MAYKOT, J. K.; RUPP, R. F.; GHISI, E. Assessment of Gender on Requirements for Thermal Comfort in Office Buildings Located in the Brazilian Humid Subtropical Climate. Energy and Buildings, v. 158, p. 1.170-1.183, 2018.

MOZAFFARIEH, M. et al. Thermal Discomfort With Cold Extremities in Relation to Age, Gender, and Body Mass Index in a Random Sample of a Swiss Urban Population. Population Health Metrics, v. 8, n. 1, p. 17, 2010.

NICOL, J. F.; HUMPHREYS, M. A. Adaptive Thermal Comfort and Sustainable Thermal Standards for Buildings. Energy and Buildings, v. 34, n. 6, p. 563-572, 2002.

RUPP, R. F. et al. Conforto Térmico Humano em Escritórios Com Sistema Central de Condicionamento Artificial em Clima Subtropical Úmido: estudos de campo vs. abordagem analítica. Ambiente Construído, Porto Alegre, v. 17, n. 1, p. 111-123, jan./mar. 2017.

RUPP, R. F.; GHISI, E. Predicting Thermal Comfort in Office Buildings in a Brazilian Temperate and Humid Climate. Energy and Buildings v. 144. p. 152-166, 2017.
VAN MARKEN LICHTENBELT, W. D.; KINGMA, B. R. Building and Occupant Energetics: a physiological hypothesis. Architectural Science Review, v. 56, n. 1, p. 4853, fev. 2013.

VECCHI, R. Avaliação de Conforto Térmico em Edificações Comerciais que Operam sob Sistemas Mistos de Condicionamento Ambiental em Clima Temperado e Úmido. Florianópolis, 2015. Tese (Doutorado em Engenharia Civil) - Departamento de Engenharia Civil, Universidade Federal de Santa Catarina, Florianópolis, 2015.

VECCHI, R. et al. Thermal Comfort in Office Buildings: findings from a field study in mixedmode and fully-air conditioning environments under humid subtropical conditions. Building and Environment, v. 123, p. 672-683, 2017.

VESELÝ, M.; ZEILER, W. Personalized Conditioning and Its Impact on Thermal Comfort and Energy Performance: a review. Renewable and Sustainable Energy Reviews, v. 34, p. 401408, 2014.

WIJERS, S. L. J.; SARIS, W. H. M.; VAN MARKEN LICHTENBELT, W. D. Cold-Induced Adaptive Thermogenesis in Lean and Obese. Obesity, v. 18, n. 6, p. 1092-9, 2010.

WORLD HEALTH ORGANIZATION. BODY

Mass Index: BMI. Disponível em:

<http://www.who.int>. Acesso em: 21 fev. 2017.

ZHANG, H.; ARENS, E.; ZHAI, Y. A Review of the Corrective Power of Personal Comfort Systems in Non-Neutral Ambient Environments. Building and Environment, v. 91, p. 15-41, 2015.

\section{Agradecimentos}

Os autores agradecem a CAPES e ao CNPq pelos recursos investidos nessa pesquisa. 


\section{Matheus de Cezaro Menegatti}

Laboratório de Eficiência Energética em Edificações | Universidade Federal de Santa Catarina | Trindade | Florianópolis - SC - Brasil | Caixa Postal 476 | CEP 88040-970 | Tel.: (48) 3721-5184 | E-mail: menegatti_81@hotmail.com

\section{Ricardo Forgiarini Rupp}

Laboratório de Eficiência Energética em Edificações | Universidade Federal de Santa Catarina | E-mail: ricardorupp@gmail.com

\section{Enedir Ghisi}

Departamento de Engenharia Civil, Centro Tecnológico, Laboratório de Eficiência Energética em Edificações | Universidade Federal de Santa Catarina | Tel.: (48) 3721-2115 | E-mail: enedir.ghisi@ufsc.br

Revista Ambiente Construído

Associação Nacional de Tecnologia do Ambiente Construído

Av. Osvaldo Aranha, 99 - 3o andar, Centro

Porto Alegre - RS - Brasil

CEP $90035-190$

Telefone: +55 (51) 3308-4084

Fax: +55 (51) 3308-4054

www. seer. ufrgs. br/ ambienteconstruido

E-mail: ambienteconstruido@ufrgs.br

(c) (1) This is an open-access article distributed under the terms of the Creative Commons Attribution License. 